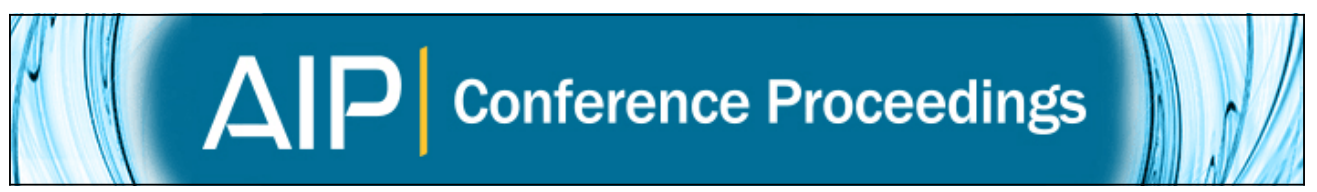

\title{
Universal oscillations of high-order cumulants
}

Christian Flindt, Christian Fricke, Frank Hohls, Tomáš Novotný, Karel Netočný, Tobias

Brandes, and Rolf J. Haug

Citation: AIP Conference Proceedings 1129, 453 (2009); doi: 10.1063/1.3140497

View online: http://dx.doi.org/10.1063/1.3140497

View Table of Contents:

http://scitation.aip.org/content/aip/proceeding/aipcp/1129?ver=pdfcov

Published by the AIP Publishing

\section{Articles you may be interested in}

Cryogenic amplifier for shot noise measurement at $20 \mathrm{mK}$

Appl. Phys. Lett. 103, 172104 (2013); 10.1063/1.4826681

High-order cumulants in the counting statistics of asymmetric quantum dots

Appl. Phys. Lett. 96, 202103 (2010); 10.1063/1.3430000

Magnetoasymmetric noise in an Aharanov-Bohm interferometer

AIP Conf. Proc. 1129, 435 (2009); 10.1063/1.3140493

Asymmetry of the excess finite-frequency noise

AIP Conf. Proc. 1129, 431 (2009); 10.1063/1.3140492

Spin oscillation of a quantum dot embedded in a ferromagnetic ring with two interacting electrons

J. Appl. Phys. 99, 08F711 (2006); 10.1063/1.2177410 


\title{
Universal oscillations of high-order cumulants
}

\author{
Christian Flindt ${ }^{* \dagger}$, Christian Fricke**, Frank Hohls**, Tomáš Novotný ${ }^{*}$, \\ Karel Netočný $y^{\ddagger}$, Tobias Brandes ${ }^{\S}$ and Rolf J. Haug ${ }^{* *}$ \\ ${ }^{*}$ Department of Physics, Harvard University, 17 Oxford Street, Cambridge, MA 02138, USA \\ ${ }^{\dagger}$ Department of Condensed Matter Physics, Faculty of Mathematics and Physics, Charles \\ University, Ke Karlovu 5, 12116 Prague, Czech Republic \\ ** Institut für Festkörperphysik, Leibniz Universität Hannover, D 30167 Hannover, Germany \\ ${ }^{\ddagger}$ Institute of Physics AS CR, Na Slovance 2, 18221 Prague, Czech Republic. \\ $\S$ Institut für Theoretische Physik, Technische Universität Berlin, D 10623 Berlin, Germany.
}

\begin{abstract}
We discuss our recent measurement of high-order cumulants of charge transport through a quantum dot. The cumulants were found to oscillate as functions of measurement time before reaching their linear-in-time asymptotics. A theoretical analysis revealed that such oscillations in fact constitute a universal phenomenon: for a large class of stochastic processes the high-order cumulants are predicted to oscillate as functions of basically any parameter. Here, we give an overview of these recent results, provide an outlook on future applications of our findings, and formulate a number of open questions.
\end{abstract}

Keywords: Counting statistics, cumulants, universal oscillations

PACS: 02.50.Ey, 72.70.+m, 73.23.Hk

Introduction. Fluctuations of the electrical current through nano-scale conductors have become a popular tool for characterizing and understanding stochastic charge transport processes $[1,2]$. In particular, the cumulants of the distribution of transferred charges are believed to contain detailed information about the microscopic mechanisms underlying the stochastic process. While the field, commonly known as counting statistics, so far has been dominated by theoretical studies, a number of recent experiments have brought this picture into a more balanced shape $[3,4,5,6,7]$. Experimental studies of the cumulants require high-quality statistics, which only now is becoming available via refined measurement techniques.

Very recently we studied experimentally and theoretically the cumulants of the number of charges tunneling through a quantum dot [8]. Experimentally, we found that the high-order cumulants oscillated as functions of time before reaching their linear-in-time asymptotics. From a theoretical analysis we were able to show that such oscillations in fact constitute a universal phenomenon: for a large class of stochastic processes the high-order cumulants become oscillatory functions of basically any parameter. This explained the measured oscillations of the high-order cumulants as functions of time as well as previous theoretical studies, where high-order cumulants in very different systems had been found to oscillate as functions of certain parameters $[9,10,11,12]$.

In this contribution we give an overview of our experimental findings and the theory underlying the universal oscillations of high-order cumulants. We discuss the general applicability of our theory, show how cumulant oscillations can be used to extract information about the cumulant generating function, and formulate a number of open questions. For further details of our work we refer the interested reader to Ref. [8].

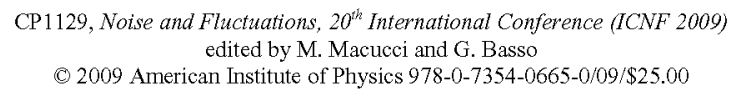



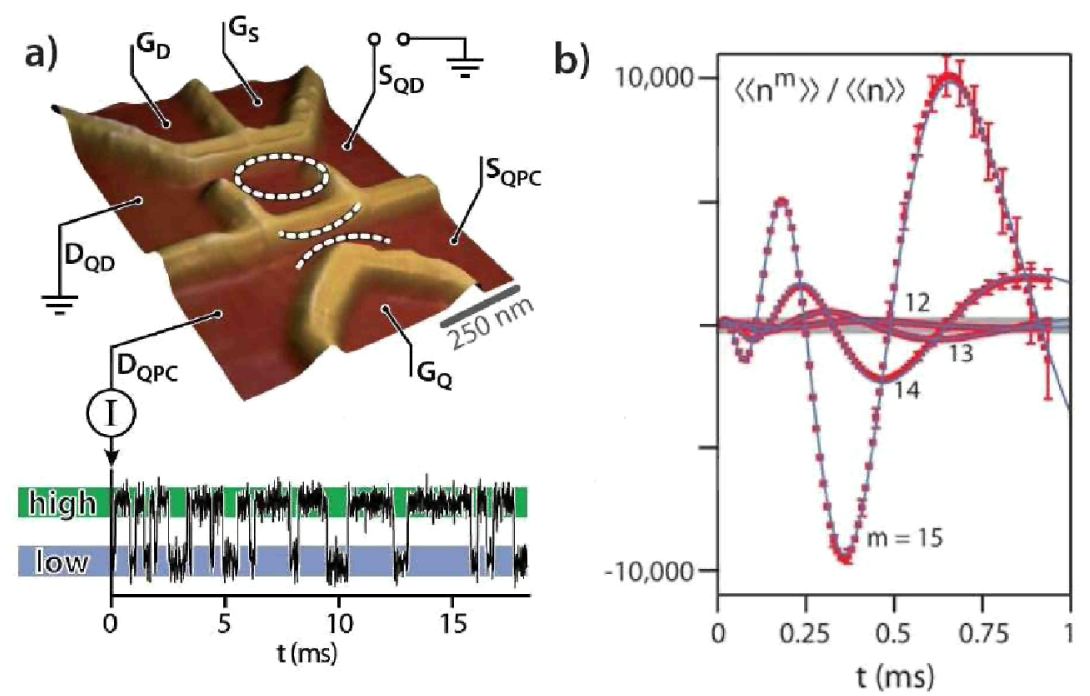

FIGURE 1. Experimental setup and results. a) Atomic force microscope topography of quantum dot (QD, dashed ring) and quantum point contact (QPC, dashed lines). Single electrons enter the QD from the source $\left(S_{\mathrm{QD}}\right)$ at rate $\Gamma_{S}=1.46 \mathrm{kHz}$ and leave via the drain at rate $\Gamma_{D}=2.97 \mathrm{kHz}$. Only a single electron at a time is allowed to occupy the QD. The current running through the QPC switches back and forth between a high and a low level as an electron enters and leaves the QD. This allows us to count the number of electrons that have passed through the QD in real-time. A typical time-trace of the QPC current is shown. b) Results for the high-order cumulants $\left\langle\left\langle n^{m}\right\rangle\right\rangle$ as functions of time. The experimental results are indicated with dots, while the full lines show rate equation calculations. The error bars were obtained from numerical simulations of the experiment. Figure adopted from Ref. [8]

Experiment. Our experimental setup is shown in Fig. 1a. Here, electrons are driven through a quantum dot (dashed ring) operated in the Coulomb-blockade regime, where only a single additional electron at a time is allowed to occupy the dot. A large bias across the dot ensures that the charge transport is unidirectional. Electrons enter the dot with rate $\Gamma_{S}$ from the source electrode $\left(S_{\mathrm{QD}}\right)$ and leave with rate $\Gamma_{D}$ via the drain $\left(D_{\mathrm{QD}}\right)$. A nearby quantum point contact (dashed lines) is used to count the number of electrons tunneling through the dot: a separate current running through the quantum point contact is suppressed every time an electron enters the dot. The suppression is lifted as the electron leaves the dot via the drain electrode. From the switches of the current through the quantum point contact it is thus possible to count the number of electrons passing through the dot. By counting a large number of tunneling events we were able to determine the distribution of the number of transferred charges $P_{n}(t)$ during the time span $[0, t]$ and in particular the cumulants of the distribution $\left\langle\left\langle n^{m}\right\rangle\right\rangle, m=1,2,3, \ldots$. The first cumulant is the mean $\langle\langle n\rangle\rangle=\langle n\rangle$, the second is the variance $\left\langle\left\langle n^{2}\right\rangle\right\rangle=\left\langle n^{2}\right\rangle-\langle n\rangle^{2}$, and the third is the skewness $\left\langle\left\langle n^{3}\right\rangle\right\rangle=\left\langle(n-\langle n\rangle)^{3}\right\rangle$. The general definition is given below.

Our experimental results for the high-order cumulants $\left\langle\left\langle n^{m}\right\rangle\right\rangle$ are shown in Fig. 1b. Most notably, the cumulants oscillate as functions of time before reaching their linear- 
in-time asymptotics (not shown). Moreover, the magnitudes of the high-order cumulants grow significantly compared to the first few cumulants which are typically on the order of unity when normalized with respect to the first cumulant. Below, we argue that oscillations and factorial growth of cumulants in fact constitute a universal phenomenon.

Theory. The following analysis applies to a large class of stochastic processes and it is not essential whether the underlying physics is classical or quantum mechanical. Given a distribution function $P_{n}(t)$, the cumulants are defined as derivatives of the cumulant generating function (CGF) $S(z, \lambda)$ with respect to the counting field $z$ at zero, $\left.\left\langle\left\langle n^{m}\right\rangle\right\rangle \equiv \partial_{z}^{m} S(z, \lambda)\right|_{z \rightarrow 0}$. Here, $\lambda$ denotes the set of all parameters of the stochastic transport process, including measurement time, and the CGF corresponding to $P_{n}(t)$ is

$$
S(z, \lambda) \equiv \ln \sum_{n} P_{n}(t) e^{n z}
$$

In order to understand the experimentally observed oscillations of the high-order cumulants with time we study the singularities of the CGF in the complex $z$-plane. As recently discussed by M. V. Berry, the high-order derivatives of a function are intimately linked with the singularities of the function [13]. We thus consider the generic situation where the CGF has a number of singularities $z, j=1,2,3, \ldots$ in the complex plane. These can be finite-order poles or branch-points. Exceptions to this scenario do exist, e.g. the Poission process whose CGF is an entire function.

Close to a singularity $z \simeq z_{j}$ we can approximate the CGF as $S(z, \lambda) \simeq A_{j} /\left(z-z_{j}\right)^{\mu_{j}}$ for some $A_{j}$ and $\mu_{j}$. For a finite-order pole, $\mu_{j}$ would be the order of the pole. Taking derivatives with respect to the counting field $z$, we find $\partial_{z}^{m} S(z, \lambda) \simeq(-1)^{m} A_{j} B_{m, \mu_{j}} /(z-$ $\left.z_{j}\right)^{m+\mu_{j}}$ with $B_{m, \mu_{j}}=\left(\mu_{j}+m-1\right)\left(\mu_{j}+m-2\right) \ldots \mu_{j}$. At first, this approximation of the derivatives close to the singularity $z_{j}$ may not seem very useful, since the cumulants are defined as the derivatives of the CGF at $z=0$. However, according to Darboux's theorem [13], the approximation of the derivative becomes better away from $z \simeq z_{j}$ with increasing order $m$. This allows us to express the high-order cumulants as a sum of contributions from all singularities, i.e.

$$
\left\langle\left\langle n^{m}\right\rangle\right\rangle \rightarrow \sum_{j}(-1)^{\mu_{j}} A_{j} B_{m, \mu_{j}} e^{i\left(m+\mu_{j}\right) \arg z_{j}} /\left|z_{j}\right|^{m+\mu_{j}}
$$

having introduced the polar coordinates $z_{j}=\left|z_{j}\right| e^{i \arg z_{j}}$. The singularities come in complex-conjugate pairs, ensuring that the cumulants evaluate to real numbers.

We are now ready to discuss a number of ubiquitous features of high-order cumulants. First, we note that the high-order cumulants generally grow factorially in magnitude with the order $m$ due to the factors $B_{m, \mu_{j}}$. Secondly, we notice that the singularities closest to $z=0$ dominate at large orders and contributions from other singularities can thus be neglected for large enough $m$. Finally, we see that the high-order cumulants become oscillatory functions of any parameter among $\lambda$ that changes the position of the dominating singularities due to the factor $e^{i\left(m+\mu_{j}\right) \arg z_{j}}$. The situation becomes particularly simple if only a single complex-conjugate pair of singularities is closest to $z=0$. Denoting this pair, the corresponding expansion coefficients, and the factorial factor by $\bar{z}, \bar{z}^{*}, \bar{\mu}, \bar{A}$, and $\bar{B}_{m}$, respectively, the high-order cumulants become

$$
\left\langle\left\langle n^{m}\right\rangle\right\rangle \rightarrow(-1)^{\bar{\mu}} 2 \bar{A} \bar{B}_{m} \cos [(m+\bar{\mu}) \arg \bar{z}] /|\bar{z}|^{m+\bar{\mu}}
$$


and we clearly see the oscillatory behavior. Returning to our experiment in Fig. 1, we can now understand the nature of the observed oscillations of the high-order cumulants with time. A pair of complex-conjugate singularities moves in the complex plane as functions of time, thereby changing arg $\bar{z}$, and thus making the high-order cumulants oscillate. The large magnitude of the oscillations is due to the factor $\bar{B}_{m}$. Here, we have not given the explicit expression for the CGF relevant to our experimental setup or the analysis of the corresponding singularities and their parameter dependencies. For such details, we refer the interested reader to Ref. [8]. We note that previous theoretical studies of different systems showing oscillations of high-order cumulants as functions of certain parameters $[9,10,11,12]$ may equally well be explained within the same theoretical framework.

Experimentally, oscillations of the high-order cumulants can be used to extract information about the CGF describing the charge transfer process. For a fixed set of parameters $\lambda$, the cumulants are predicted to oscillate as functions of the cumulant order $m$ with the frequency given by the angle $\arg \bar{z}$ of the singularity $\bar{z}$ with the real-axis, see Eq. (3). This can be used to locate the dominating singularities of the CGF: by measuring the cumulants as functions of the cumulant order $m$, the angle $\arg \bar{z}$ can be extracted from the frequency of the oscillations, while the argument $|\bar{z}|$ enters the magnitude of the oscillations. Additionally, the nature of the singularity, e.g. the order of a pole $\bar{\mu}$, determines the phase of the oscillations and should thus be experimentally detectable.

Conclusions. We have presented an experimental and theoretical study of high-order cumulants of charge transport through a quantum dot and shown that oscillations of high-order cumulants constitute a universal phenomenon for a large class of stochastic processes. Some open questions remain and deserve further investigation: What is the physical origin of the oscillations? What information is generally available in high-order cumulants? What do the oscillations tell us about a particular system?

Acknowledgements. The work was supported by the Villum Kann Rasmussen Foundation, the Czech Science Foundation via grant 202/07/J051, BMBF via nanoQuit, DFG via QUEST, research plan MSN 0021620834 financed by the Ministry of Education of the Czech Republic, and DFG project BR 1528/5-1.

\section{REFERENCES}

1. Ya, M. Blanter and M. Büttiker, Phys. Rep. 336, 1 (2000)

2. Quantum Noise in Mesoscopic Physics, edited by Yu. V. Nazarov (Kluwer, Dordrecht, 2003)

3. B. Reulet, J. Senzier, and D. E. Prober, Phys. Rev. Lett. 91, 196601 (2003)

4. Yu. Bomze et al., Phys. Rev. Lett. 95, 176601 (2005)

5. S. Gustavsson et al., Phys. Rev. Lett. 96, 076605 (2006)

6. T. Fujisawa, T. Hayashi, R. Tomita, and Y. Hirayama, Science 312, 1634 (2006)

7. A. V. Timofeev et al., Phys. Rev. Lett. 98, 207001 (2007)

8. C. Flindt et al., arXiv:0901.0832 (2009)

9. S. Pilgram and M. Büttiker, Phys. Rev. B 67, 235308 (2003)

10. H. Förster, S. Pilgram, and M. Büttiker, Phys. Rev. B 72, 075301 (2005)

11. H. Förster, P. Samuelsson, S. Pilgram, and M. Büttiker, Phys. Rev. B 75, 035340 (2007)

12. C. Flindt et al., Phys. Rev. Lett. 100, 150601 (2008)

13. M. V. Berry, Proc. R. Soc. A 461, 1735 (2005) 